\title{
Synergistic effect of sorafenib and cGvHD in patients with high-risk FLT3-ITD+AML allows long-term disease control after allogeneic transplantation
}

\author{
A. Tschan-Plessl ${ }^{1}$ • J. P. Halter ${ }^{1}$ • D. Heim ${ }^{1}$ - M. Medinger ${ }^{1}$ - J. R. Passweg ${ }^{1} \cdot$ S. Gerull ${ }^{1}$
}

Received: 20 April 2015 / Accepted: 15 July 2015 /Published online: 2 August 2015

(C) Springer-Verlag Berlin Heidelberg 2015

\begin{abstract}
The multikinase inhibitor sorafenib has shown a strong anti-leukemic effect in FMS-like tyrosine kinase 3 (FLT3)-mutated acute myeloid leukemia (AML); however, remission is often transient. To better understand the role of sorafenib, we performed a retrospective analysis of all patients who received sorafenib in combination with allogeneic hematopoietic stem cell transplantation (HSCT) at our center. Seventeen patients with FLT3-ITD positive AML were treated with sorafenib in combination with allogeneic HSCT. Seven patients received sorafenib therapy pre- and posttransplant, and $10 \mathrm{pa}-$ tients were given sorafenib only posttransplant. Median duration of sorafenib treatment was 13 months (range 1-42); median dose was $600 \mathrm{mg}$ (range 100-1200). Fourteen patients $(82 \%)$ achieved a complete remission (CR), while 5 patients (29\%) eventually developed progressive disease. Developing chronic graft-versus-host disease (GvHD) had a strong protective influence on the risk of sorafenib resistance $(p=0.028$, HR $0.08,95 \%$ CI $0.01-0.76$ ). In a total of 8 patients, sorafenib had to be stopped, paused or dose-reduced due to toxicity. In 5 patients with pronounced toxicity, we switched to an alternating dosing schedule with 1 month on/1 month off sorafenib. These patients subsequently remained in sustained complete molecular remission, with a median follow-up of 20 months. Our data indicate that sorafenib can achieve high rates of sustained remission in high-risk patients treated in combination with HSCT.
\end{abstract}

Keywords flt3 · Tyrosine-kinase inhibitor · Sorafenib ·

Allogeneic

\section{A. Tschan-Pless1}

astrid.plessl@usb.ch

1 Department of Hematology, University Hospital Basel, Petersgraben 4, 4031 Basel, Switzerland

\section{Introduction}

Acute myeloid leukemia (AML) is a heterogeneous disease with large variations in prognosis depending on cytogenetic and molecular characteristics $[1,2]$. Mutations in the FMSlike tyrosine kinase 3 (FLT3) are one of the most common molecular alterations observed in AML and have been associated with a poor prognosis [3, 4].

Tyrosine-kinase inhibitors (TKI) have shown activity in FLT3 mutated AML [5-7]; however, remissions are generally short, and resistance develops in the majority of patients. Randomized studies of TKIs in combination with chemotherapy have failed to show an improved outcome $[8,9]$. Recent data suggest that longterm remissions can be achieved with sorafenib monotherapy when given after allogeneic transplant $[10,11]$; however, other studies failed to observe such a synergistic effect [12].

To better understand the role of sorafenib in the treatment of FLT3-mutated AML, we retrospectively analyzed all patients treated with sorafenib in conjunction with allogeneic transplant at our institution.

\section{Patients and methods}

\section{Study design}

We performed a retrospective analysis of all patients with FLT3-ITD positive AML that were treated with sorafenib after allogeneic transplant at the University Hospital Basel until March 2014. All patients gave written informed consent to the analysis of outcome data and the study was performed according to the regulations of the local ethics committee: "Ethikkommission Nordwest- und Zentralschweiz".

Variables were collected from chart review including patient characteristics (age at diagnosis and transplant, sex, 
CMV status), disease characteristics (FAB and WHO classification, cytogenetics, FLT3 mutation status, presence of other molecular markers), treatment and response to them including sorafenib treatment duration and dosing, side effects and dose reduction or interruptions, transplant characteristics including type of conditioning, and graft-versus-host disease (GvHD) prophylaxis, donor type, HLA-match, stem cell source, and grading of GvHD.

\section{Definitions}

Karyotype risk group was categorized according to SWOG criteria [13]. Non-myeloablative and myeloablative conditioning were defined according to published criteria [14]. Acute GVHD (aGVHD) and chronic GVHD (cGVHD) were graded according to standard criteria $[15,16]$. Treatment response was defined according to Cheson criteria [17]. Time to treatment failure was defined as the interval between start of sorafenib treatment and sorafenib resistance. Sorafenib resistance was defined as morphologic or molecular relapse or progression (detection or increase of blasts in peripheral blood or bone marrow or extramedullary disease manifestations, detection or increase of FLT3-ITD or - in the case of FLT3-ITD/ NPM1-positive leukemia-NPM1 copies in blood or bone marrow, assessed by quantitative PCR).

\section{Statistical analysis}

Patient and transplant characteristics were compared using Pearson's chi-square test for categorical variables. The Kaplan-Meier method was used to estimate overall survival (OS) and progression-free survival (PFS). A time-dependent Cox-regression model was used to analyze the effect of GvHD on the risk of sorafenib treatment failure.

\section{Results}

\section{Patient characteristics}

A total of 17 patients with FLT3-ITD positive AML were treated with sorafenib after allogeneic HSCT between August 2009 and March 2014 at the University Hospital Basel. Seven of these had also received sorafenib pretransplant. Follow-up was calculated as of November 1, 2014. Patient pretreatment and transplant characteristics are described in Table 1. Characteristics did not differ between patients with and without sorafenib resistance (data not shown). Median age was 53 years. More males than females were treated (11 versus 6 individuals).

\section{Induction therapy}

Standard induction therapy consisted of a first cycle of idarubicin and cytarabine and a second cycle of amsacrine and high-dose cytarabine. For reinduction, mostly high-dose cytarabine-based schemes were used (as monotherapy or in combination with mitoxantrone, clofarabine, fludarabine/ idarubicin, or cladribine/idarubicin). One patient received a combination of etoposide and mitoxantrone.

\section{Sorafenib treatment}

Characteristics of sorafenib treatment are shown in Table 2 and in Fig. 1. Ten patients received sorafenib posttransplant, five because of hematological relapse - one of them with chemotherapy-refractory relapse - three because of molecular relapse or molecular disease persistence after transplantation, and two as maintenance therapy in complete molecular remission because of disease persistence pretransplant. Eight of these 10 patients received sorafenib monotherapy, one who was refractory to chemotherapy received concurrent donor lymphocyte infusion (DLI) and 1 patient was given concomitant low dose cytarabine subcutaneously after emergence of sorafenib treatment failure. Seven patients received sorafenib pre- and posttransplant. Pretransplant indication was chemorefractory relapse in 5 patients (two after autologous transplant) and molecular persistence in 2 patients. Reasons for continued treatment posttransplant were maintenance in three, molecular relapse in two that received sorafenib in combination with DLI, and hematological relapse in 2 patients, one of which received concurrent chemotherapy.

Median duration of sorafenib treatment was 13 months (range 1-42). Treatment was started at a median of 64 days posttransplant (range 3-709 days).

Median follow-up time after initiation of sorafenib therapy was 29 months (range 14-46).

\section{Dose and toxicity}

Target dose of sorafenib was $800 \mathrm{mg}$ daily but was reduced to $100-600 \mathrm{mg}$ or transiently stopped due to toxicity in a total of seven patients. Hence, the median dose was $600 \mathrm{mg}$ (range $100-1200 \mathrm{mg}$ ). Sorafenib was discontinued in one patient due to severe diarrhea and exanthema. Toxicity leading to dose reduction or transient stop of therapy was diarrhea $(n=4)$, nausea/vomitus $(n=2)$, cytopenias $(n=1)$, hepatotoxicity $(n=$ $2)$, hand-foot syndrome $(n=1)$, and exanthema $(n=1)$. In several patients, a combination of multiple toxicities led to dose reduction; therefore, listed toxicities add up to more than $100 \%$. Other toxicities observed included glossalgia, headache, rhagades, and muscle spasms. In five patients who had been on sorafenib after allogeneic transplant for a median of 15 (range 4-20) months and were in complete molecular 
Table 1 Patient pretreatment and transplant characteristics

\begin{tabular}{|c|c|c|c|}
\hline & $\begin{array}{l}\text { All patients } \\
n=17\end{array}$ & $\begin{array}{l}\text { Patients with } \\
\text { sorafenib resistance } \\
n=6\end{array}$ & $\begin{array}{l}\text { Patients without } \\
\text { sorafenib resistance } \\
n=11\end{array}$ \\
\hline Median age at diagnosis (range) & $53(23-68)$ & $58(40-64)$ & $49(23-68)$ \\
\hline Number of females $(\%)$ & $6(35)$ & $2(33)$ & $4(36)$ \\
\hline \multicolumn{4}{|l|}{ Disease characteristics (\%) } \\
\hline FLT3-ITD mutated & $6(35)$ & $2(33)$ & $4(36)$ \\
\hline NPM1/FLT3-ITD mutated & $10(59)$ & $4(67)$ & $6(55)$ \\
\hline CEBPA/FLT3-ITD mutated & $1(6)$ & 0 & $1(9)$ \\
\hline \multicolumn{4}{|l|}{ Karyotype risk group ${ }^{\mathrm{a}}(\%)$} \\
\hline Favorable & 0 & 0 & 0 \\
\hline Intermediate & $14(82)$ & $5(83)$ & $9(82)$ \\
\hline Unfavorable & $2(12)$ & $1(17)$ & $1(9)$ \\
\hline Unknown & $1(6)$ & 0 & $1(9)$ \\
\hline \multicolumn{4}{|l|}{ Donor type (\%) } \\
\hline HLA-identical sibling & $8(47)$ & $2(33)$ & $6(55)$ \\
\hline Matched unrelated donor & $6(35)$ & $2(33)$ & $4(36)$ \\
\hline Mismatched unrelated donor & $2(12)$ & $2(33)$ & 0 \\
\hline Mismatched cord blood & $1(6)$ & 0 & $1(9)$ \\
\hline \multicolumn{4}{|l|}{ Stem cell source $(\%)$} \\
\hline PBSC & $15(88)$ & $6(100)$ & $9(82)$ \\
\hline $\mathrm{BM}$ & $1(6)$ & 0 & $1(9)$ \\
\hline $\mathrm{CB}$ & $1(6)$ & 0 & $1(9)$ \\
\hline \multicolumn{4}{|l|}{ Conditioning regimen (\%) } \\
\hline Myeloablative & $15(88)$ & $5(83)$ & $10(91)$ \\
\hline Non-Myeloablative & $2(12)$ & $1(17)$ & $1(9)$ \\
\hline \multicolumn{4}{|l|}{ GvHD prophylaxis (\%) } \\
\hline Cyclosporin A/Methotrexat & $10(59)$ & $2(33)$ & $8(73)$ \\
\hline Cyclosporin A/Methotrexat/ATG & $4(24)$ & $3(50)$ & $1(9)$ \\
\hline Cyclosporin A/Mycofenolat & $2(12)$ & $1(17)$ & $1(9)$ \\
\hline Cyclosporin A/ATG & $1(6)$ & 0 & $1(9)$ \\
\hline
\end{tabular}

${ }^{\text {a }}$ According to SWOG criteria

remission, continuous therapy was switched to a 1 month on/ 1 month off sorafenib schedule due to toxicity (headache $(n=$ $1)$, diarrhea $(n=4)$, and hand-foot syndrome $(n=2)$. Median duration of intermittent therapy was 16 months (range 5-21).

In 2 patients, sorafenib dose was increased to 1000 $1200 \mathrm{mg}$ per day due to insufficient serum levels. Target doses were obtained from published data $[18,19]$.

\section{Treatment response}

Best response to sorafenib was complete molecular remission (CMR) ( $n=11,3$ already being in CMR before sorafenib initiation), complete morphologic remission (CR) $(n=3)$, hematological response (HR) $(n=1)$, stable disease $(n=1)$, and progressive disease $(n=1)$. Secondary resistance developed in 5 (29\%) patients after a median of 6 (range 1.7-27) months. Survival status is shown in Table 3. At the time of analysis, 9 patients remained alive in CMR or CR, while 5 patients had died of relapse.

In a total of 4 patients who had previously been on intermittent therapy, sorafenib was discontinued after achieving a durable molecular remission of a minimum of 2 years. Progression free and overall survival from start of sorafenib therapy and from transplant are shown in Fig. 2. Two-year OS and PFS after start of sorafenib were 61 and $53 \%$, respectively, with 2-year posttransplant OS and PFS being 61 and $55 \%$, respectively.

\section{GvHD}

As shown in Table 3, $53 \%$ of patients developed aGvHD grade I-II $(n=7)$ or grade III-IV $(n=2)$. Chronic GvHD occurred in 11 patients $(65 \%)$, being mild $(n=3)$, moderate $(n=$ $6)$, or severe $(n=2)$. In patients that received sorafenib posttransplant and developed acute and/or chronic GvHD 
Table 2 Treatment characteristics

\begin{tabular}{|c|c|c|c|}
\hline & $\begin{array}{l}\text { All patients } \\
n=17\end{array}$ & $\begin{array}{l}\text { Patients with } \\
\text { sorafenib } \\
\text { resistance } \\
n=6\end{array}$ & $\begin{array}{l}\text { Patients without } \\
\text { sorafenib resistance } \\
n=11\end{array}$ \\
\hline \multicolumn{4}{|l|}{ Sorafenib start pre/post allo HSCT } \\
\hline Post $(\%)$ & $10(59)$ & $2(33)$ & $8(73)$ \\
\hline Pre+post $(\%)$ & $7(41)$ & $4(67)$ & $3(27)$ \\
\hline Median number of CTx cycles ${ }^{\mathrm{a}}$ pre sorafenib (range) & $2(1-5)$ & $2.5(2-5)$ & $2(2-3)$ \\
\hline \multicolumn{4}{|l|}{ Indication for sorafenib ${ }^{b}(\%)$} \\
\hline Refractory leukemia to chemotherapy & $4(24)$ & $3(50)$ & $1(9)$ \\
\hline Hematological relapse & $7(41)$ & $3(50)$ & $4(36)$ \\
\hline Molecular relapse/persistence & $4(24)$ & 0 & $4(36)$ \\
\hline Maintenance therapy in CMR & $2(12)$ & 0 & $2(18)$ \\
\hline \multicolumn{4}{|l|}{ Therapy details (\%) } \\
\hline Sorafenib monotherapy & $14(82)$ & $4(67)$ & $10(91)$ \\
\hline Combination with DLI & $1(6)$ & 0 & $1(9)$ \\
\hline Combination with CTx & $1(6)$ & $1(17)$ & 0 \\
\hline Combination with DLI and CTx & $1(6)$ & $1(17)$ & 0 \\
\hline
\end{tabular}

$D L I$ donor lymphocyte infusion, $C T x$ chemotherapy

${ }^{\text {a }}$ Each chemotherapy cycle is counted. E.g., 2 cycles of induction therapy and 1 cycle of consolidation therapy counts as 3 cycles

${ }^{\mathrm{b}}$ For patients who received sorafenib pre- and posttransplant, only pretransplant indication was counted

$(n=14)$, GvHD developed before the start of sorafenib in 8 patients. All patients that had a sustained remission without secondary resistance had concurrent cGvHD, while only one of the 6 patients with sorafenib treatment failure had chronic GvHD $(p=0.021)$. To exclude selection bias (patients with early death may not have had sufficient follow-up to develop GvHD), results were confirmed by performing a Coxregression analysis of the effect of chronic GvHD as a timedependent variable on the risk of sorafenib resistance. Including only patients that received sorafenib posttransplant; developing chronic GvHD had a strong protective influence on the risk of sorafenib resistance $(p=0.028$, HR $0.08,95 \%$ CI 0.01-0.76).

Standard GvHD treatment consisted of systemic and topical steroids as well as calcineurin inhibitor therapy. In cases of refractory GvHD, individual treatment approaches such as photopheresis, methotrexate, or imatinib were applied.

\section{Discussion}

We describe here a series of 17 patients with FLT3-ITD mutated AML who were treated with sorafenib as well as allogeneic stem cell transplantation. We observed a high rate of sustained responses, and it is remarkable that all patients who remained in sustained remission had concurrent cGvHD, suggesting a synergistic effect.

Previous reports of sorafenib in conjunction with allogeneic transplant have shown mixed results. The largest patient group was described by Metzelder et al. who reported on 65 patients, 29 of which had received an allogeneic transplant [10]. Transplant patients had a lower rate of sorafenib resistance (38 versus $47 \%$ ) and a longer time to development of resistance (197 versus 136 days). In a recent phase I study by Chen et al., 22 patients were treated with sorafenib as maintenance after allogeneic transplant, with relapse observed in only 2 patients after a median follow-up of 16 months [20]. Conversely, Sharma et al. reported on 16 patients who received sorafenib for posttransplant relapse, with $(n=8)$ or without $(n=8)$ additional chemotherapy, and observed only partial remission in 3 patients, with no cases of complete remission [12]. Reasons for these comparatively poor results might have been the non-continuous dosing used, as well as particularly advanced disease in most patients.

Reports on rates of GvHD in patients treated with sorafenib are also mixed. While Metzelder et al. observed GvHD in only 7 of 65 patients, others have suspected that sorafenib actually provokes GvHD [21]. Besides observing chronic GvHD in 4 of 7 patients treated with sorafenib, Yokoyama et al. also provide murine data suggesting that sorafenib might exacerbate GvHD. Other small case series have described worsening of GvHD with sorafenib [11, 22], while the two larger patient cohorts described by Sharma et al. and Chen et al. had chronic GvHD rates of 0 and $38 \%$, respectively $[12,20]$. Reasons for these variations could be differences in timing and dosing of sorafenib treatment, concurrent immunosuppression and use of donor lymphocyte infusion, as well as stochastic variance due to small patient numbers. 


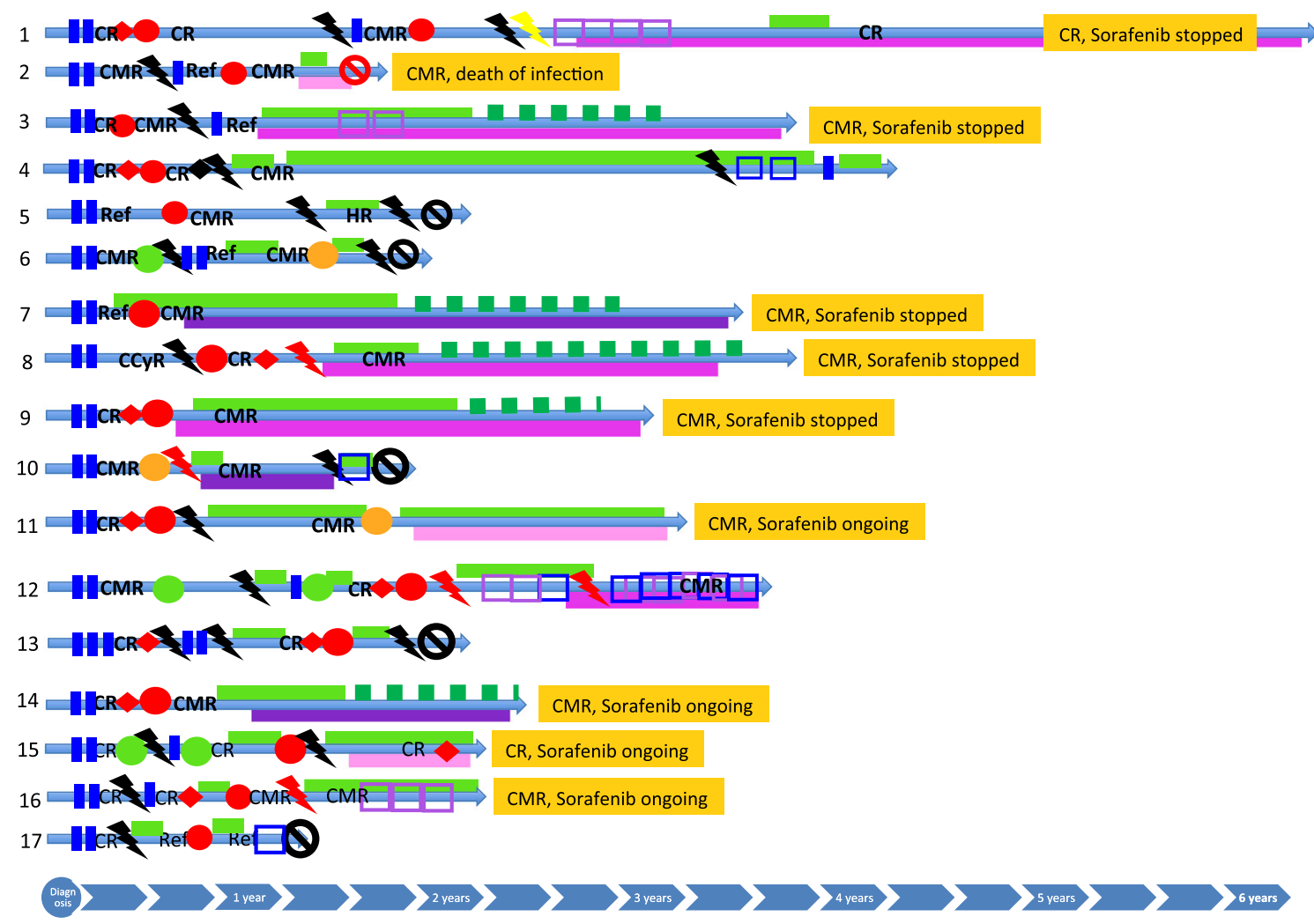

\section{Legend}

Induction chemotherapy

Allo HSCT (myeloablative conditioning)

Allo HSCT (reduced intensity conditioning)

Auto HSCT

- Hematological Relapse

- Molecular Relapse/molecular progression

- Molecular persistency

Sorafenib therapy

$\square \quad$ Donor lymphocyte infusion

$\square$ Low-dose Chemotherapy: Cytarabin or Azacitidin subcutanously

Radiation therapy of extramedullary disease manifestations

( Death of disease progression

\section{cGvHD, maximum Grading mild}

cGvHD, maximum Grading moderate

cGvHD, maximum Grading severe

Fig. 1 Illustration of therapy and outcome in individual patients

While we have observed comparatively high rates of cGvHD, the role of sorafenib in provoking or worsening GvHD remains unclear for a number of confounding factors. These include the fact that patients who were still on immunosuppression at the time of relapse or progression had rapid tapering of immunosuppressive agents; several patients received DLI and median treatment duration in our cohort was comparatively long. Unfortunately, other authors have not 
Table 3 GVHD details and survival status

\begin{tabular}{llll}
\hline & All patients & $\begin{array}{l}\text { Patients with } \\
\text { sorafenib } \\
\text { resistance } \\
n=6\end{array}$ & $\begin{array}{l}\text { Patients without } \\
\text { sorafenib } \\
\text { resistance } \\
n=11\end{array}$ \\
& $\begin{array}{l}n=17 \\
\text { Number of patients developping GvHD (\%) }\end{array}$ & & \\
aGvHD grade I-II & $7(41)$ & $3(50)$ & $4(36)$ \\
aGvHD grade III-IV & $2(12)$ & $1(17)$ & $1(9)$ \\
cGvHD, overall & $11(65)$ & $1(17)$ & $10(91)$ \\
cGvHD, mild & $3(18)$ & 0 & $3(27)$ \\
cGvHD, moderate & $6(35)$ & $1(17)$ & $5(45)$ \\
cGvHD, severe & $2(12)$ & 0 & $2(18)$ \\
Patients alive & $11(65)$ & $2(33)$ & $9(82)$ \\
Patients in CMR, sorafenib ongoing (continous therapy) & $2(12)$ & 0 & $2(18)$ \\
Patients in CMR, sorafenib ongoing (intermittent therapy) & $1(6)$ & 0 & $1(9)$ \\
Patients in CMR, sorafenib stopped & $4(24)$ & 0 & $4(36)$ \\
Patients in CR, sorafenib ongoing (continous therapy) & $1(6)$ & 0 & $1(9)$ \\
Patients in CR, sorafenib stopped & $1(6)$ & 0 & $1(9)$ \\
Patients with relapse or progression, sorafenib ongoing & $1(6)$ & $1(17)$ & 0 \\
Patients with relapse, sorafenib stopped & $1(6)$ & $1(17)$ & 0 \\
Patients dead & $6(35)$ & $4(67)$ & $2(18)$ \\
Death of disease progression & $5(29)$ & $4(67)$ & $1(9)$ \\
Death of infection in complete remission & $1(6)$ & 0 & $1(9)$ \\
Median follow-up of survivors in months (range) & $29(14-46)$ & $37(28-46)$ & $29(14-44)$ \\
\hline
\end{tabular}

${ }^{\text {a }}$ Intermittent therapy: one month on, one month off commented on the relationship between long-term remission and concurrent GvHD. Due to the fact that long-term remissions with sorafenib treatment seem to occur predominantly following allogeneic transplantation, a certain synergism can be assumed. It can only be speculated on mechanisms of this synergy, which could include activation of alloreactivity by sorafenib, a reduction of tumor mass and rate of disease evolution to facilitate the graft-versus-leukemia effect or a combination of these effects.

A further novel aspect of our data is the alternating dosing (1 month on/1 month off) that was applied in selected patients that had been in prolonged remission and suffered from toxicity of sorafenib. Due to the relatively limited experience with the use of sorafenib in AML patients, no standards exist for suggested length of treatment in patients who have attained a remission. In poor-risk patients with posttransplant relapse, the long-term remission we observed in selected patients led to the question of whether sorafenib could be stopped after a certain duration of CMR without risk of relapse. Due to significant concern on our part but also on part of the patients, we chose to deescalate treatment by first switching to alternate
Fig. 2 Overall and progression free survival
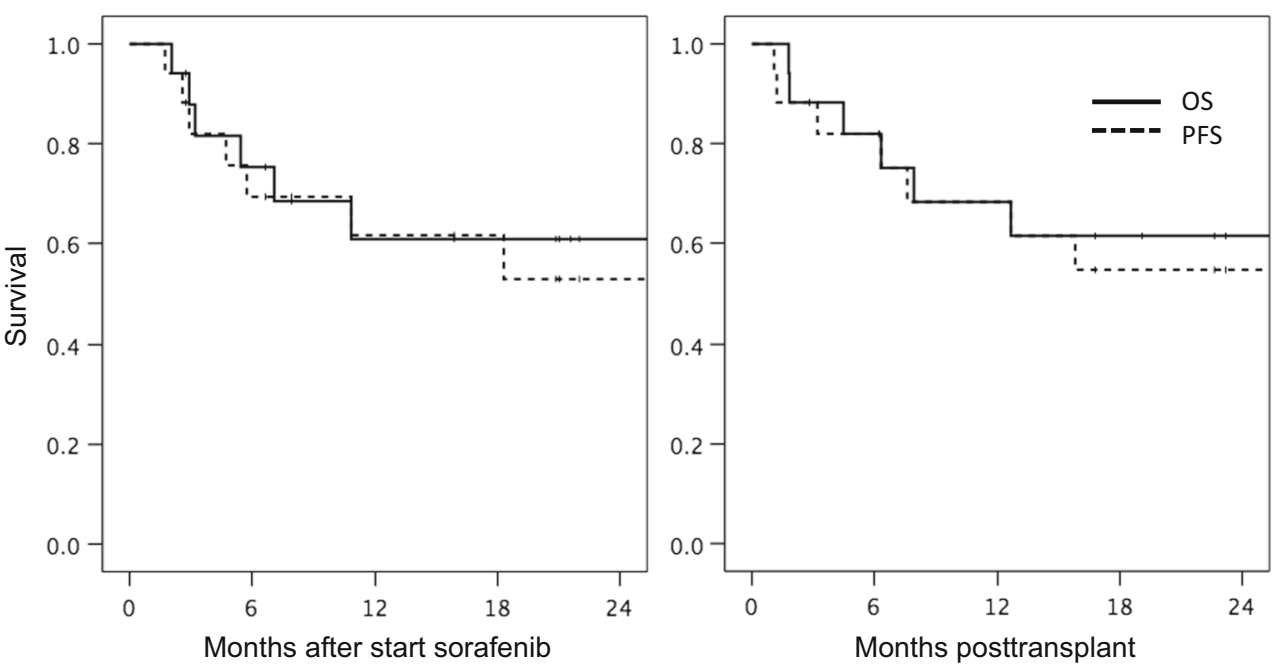
dosing. We hypothesized that the anti-leukemic effect is mainly the result of the graft-versus-leukemia effect; hence, an intermittent use of sorafenib would reduce toxicity by providing treatment free periods for patients, but continue to suppress intermittently any remaining residual disease. While no conclusions can be drawn from our small patient numbers, the fact that all patients on alternate dosing have remained in CMR between 5 and 21 months is notable. We have now stopped sorafenib completely in selected patients that have been in CMR for a minimum of 2 years.

Major drawbacks of our study include the retrospective nature and the small patient numbers, as well as the fact that the role of sorafenib versus the effect of cGvHD in maintaining long-term remission cannot be elucidated. However, the comparatively long follow-up as well as the novel aspect of alternating dosing makes the data noteworthy nonetheless. In conclusion, our results are very promising and show that sorafenib along with a probable synergistic effect of cGvHD can achieve long-term remission in high-risk patients with acceptable short-term toxicity. However, in some patients, long-term toxicity remains substantial. Prospective studies are urgently needed to determine the exact role of sorafenib in the setting of allogeneic transplant as well as to clarify optimal treatment modalities including dosage and duration.

Conflict of interest The authors declare that they have no competing interests.

\section{References}

1. Breems DA, Van Putten WL, De Greef GE et al (2008) Monosomal karyotype in acute myeloid leukemia: a better indicator of poor prognosis than a complex karyotype. J Clin Oncol 26:4791-4797

2. Burnett A, Wetzler M, Lowenberg B (2011) Therapeutic advances in acute myeloid leukemia. J Clin Oncol 29:487-494

3. Stirewalt DL, Radich JP (2003) The role of FLT3 in haematopoietic malignancies. Nat Rev Cancer 3:650-665

4. Frohling S, Schlenk RF, Breitruck J et al (2002) Prognostic significance of activating FLT3 mutations in younger adults (16 to 60 years) with acute myeloid leukemia and normal cytogenetics: a study of the AML Study Group Ulm. Blood 100:4372-4380

5. Knapper S, Burnett AK, Littlewood T et al (2006) A phase 2 trial of the FLT3 inhibitor lestaurtinib (CEP701) as first-line treatment for older patients with acute myeloid leukemia not considered fit for intensive chemotherapy. Blood 108:3262-3270

6. Fischer T, Stone RM, Deangelo DJ et al (2010) Phase IIB trial of oral Midostaurin (PKC412), the FMS-like tyrosine kinase 3 receptor (FLT3) and multi-targeted kinase inhibitor, in patients with acute myeloid leukemia and high-risk myelodysplastic syndrome with either wild-type or mutated FLT3. J Clin Oncol 28:4339-4345

7. Swords R, Freeman C, Giles F (2012) Targeting the FMS-like tyrosine kinase 3 in acute myeloid leukemia. Leukemia 26:21762185
8. Serve H, Krug U, Wagner R et al (2013) Sorafenib in combination with intensive chemotherapy in elderly patients with acute myeloid leukemia: results from a randomized, placebo-controlled trial. J Clin Oncol 31:3110-3118

9. Levis M, Ravandi F, Wang ES et al (2011) Results from a randomized trial of salvage chemotherapy followed by lestaurtinib for patients with FLT3 mutant AML in first relapse. Blood 117:3294 3301

10. Metzelder SK, Schroeder T, Finck A et al (2012) High activity of sorafenib in FLT3-ITD-positive acute myeloid leukemia synergizes with allo-immune effects to induce sustained responses. Leukemia 26:2353-2359

11. Sora F, Chiusolo P, Metafuni E et al (2011) Sorafenib for refractory FMS-like tyrosine kinase receptor-3 (FLT3/ITD+) acute myeloid leukemia after allogenic stem cell transplantation. Leuk Res 35: 422-423

12. Sharma M, Ravandi F, Bayraktar UD et al (2011) Treatment of FLT3-ITD-positive acute myeloid leukemia relapsing after allogeneic stem cell transplantation with sorafenib. Biol Blood Marrow Transplant 17:1874-1877

13. Slovak ML, Kopecky KJ, Cassileth PA et al (2000) Karyotypic analysis predicts outcome of preremission and postremission therapy in adult acute myeloid leukemia: a Southwest Oncology Group/ Eastern Cooperative Oncology Group Study. Blood 96:40754083

14. Bacigalupo A, Ballen K, Rizzo D et al (2009) Defining the intensity of conditioning regimens: working definitions. Biol Blood Marrow Transplant 15:1628-1633

15. Filipovich AH, Weisdorf D, Pavletic S et al (2005) National Institutes of Health consensus development project on criteria for clinical trials in chronic graft-versus-host disease: I. Diagnosis and staging working group report. Biol Blood Marrow Transplant 11: 945-956

16. Glucksberg H, Storb R, Fefer A et al (1974) Clinical manifestations of graft-versus-host disease in human recipients of marrow from HL-A-matched sibling donors. Transplantation 18:295-304

17. Cheson BD, Bennett JM, Kopecky KJ et al (2003) Revised recommendations of the International Working Group for Diagnosis, Standardization of Response Criteria, Treatment Outcomes, and Reporting Standards for Therapeutic Trials in Acute Myeloid Leukemia. J Clin Oncol 21:4642-4649

18. Strumberg D, Richly H, Hilger RA et al (2005) Phase I clinical and pharmacokinetic study of the Novel Raf kinase and vascular endothelial growth factor receptor inhibitor BAY 43-9006 in patients with advanced refractory solid tumors. J Clin Oncol 23:965-972

19. Fukudo M, Ito T, Mizuno T et al (2014) Exposure-toxicity relationship of sorafenib in Japanese patients with renal cell carcinoma and hepatocellular carcinoma. Clin Pharmacokinet 53:185-196

20. Chen YB, Li S, Lane AA et al (2014) Phase I trial of maintenance sorafenib after allogeneic hematopoietic stem cell transplantation for fms-like tyrosine kinase 3 internal tandem duplication acute myeloid leukemia. Biol Blood Marrow Transplant 20(12):20422048

21. Yokoyama H, Lundqvist A, Su S, Childs R (2010) Toxic effects of sorafenib when given early after allogeneic hematopoietic stem cell transplantation. Blood 116:2858-2859

22. Metzelder S, Wang Y, Wollmer E et al (2009) Compassionate use of sorafenib in FLT3-ITD-positive acute myeloid leukemia: sustained regression before and after allogeneic stem cell transplantation. Blood 113:6567-6571 